\title{
REGISTERFORSCHUNG: VERWALTUNGS- UND STATISTIKDATEN FÜR DIE WISSENSCHAFT
}

\author{
von Harald Oberhofer, Gerhard Schwarz und Michael Strassnig
}

Zusammenfassung: Die Verfügbarkeit von Daten aus der Verwaltung sowie der Bundesstatistik für die (Grundlagen-)Forschung wird zunehmend ein Faktor für die Wettbewerbsfähigkeit eines Forschungsstandortes - insbesondere in den Sozialwissenschaften aber auch der Gesundheitswissenschaft - und könnte einen wichtigen Beitrag für eine Evidenzorientierung in der Politik und Verwaltung liefern. In Österreich bildet vor allem die vergleichsweise unklare und zerklüftete Rechtslage in diesem Bereich sowie politische Widerstände gegen einen Zugang zu diesen Daten für die Wissenschaft eine wesentliche Herausforderung. Aus internationaler Perspektive ist aber die Öffnung dieser Daten geradezu notwendig und ein Gebot der Stunde.

Schlagwörter: Registerforschung; Verwaltungsdaten; Statistikdaten; DSGVO

\section{USING ADMINISTRATIVE AND OFFICIAL STATISTICS DATA FOR SCIENTIFIC RESEARCH: BENEFITS AND POLITICAL CHALLENGES}

Abstract: The availability of administrative data and official statistics data for (basic) research becomes increasingly a determinant for the competitiveness of a country's research capabilities. This is particular true for the social sciences including economics as well as health research and could provide an important contribution for evidencebased politics and public administration. In Austria, the greatest challenges are the unclear and uneven legal situation and political resistance against a more open and better structured access to registry and statistical data for science. From an international perspective, the accessibility of administrative data is self-evident and state of the art in many European countries.

Keywords: registry data; statistical data; research; GDPR

DOI: https://doi.org/10.31263/voebm.v72i2.3154

(c) Harald Oberhofer, Gerhard Schwarz, Michael Strassnig

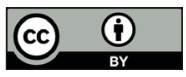

Dieses Werk ist lizenziert unter einer

Creative-Commons-Lizenz Namensnennung 4.0 International 


\section{Inhalt}

1. Einleitung

2. Demokratische Kontrolle und gesellschaftlicher Nutzen

3. Zerklüftete Rechtslage

4. Datenschutz als zentrales Anliegen

5. Die Plattform Registerforschung

\section{Einleitung}

Stellen Sie sich vor, Krebs könnte erfolgreicher als bisher bekämpft werden. Oder, dass die Wirksamkeit öffentlicher Förderungen treffsicher überprüft würde. Oder, dass es effektive Wege gäbe, Bildungskarrieren zu verfolgen und mit den gewonnenen Erkenntnissen das Schulsystem zu verbessern. Das alles ist tatsächlich möglich und noch viel mehr. Aber nicht in Österreich. Hier behindern eine restriktive Gesetzeslage sowie Widerstände aus Politik und Verwaltung den Zugang der Wissenschaft zu forschungsrelevanten Register- und Statistikdaten und damit den wissenschaftlichen Erkenntnisgewinn. In der „Plattform Registerforschung“ haben sich über 100 Wissenschaftlerinnen und Wissenschaftler zusammengefunden, um für die Wissenschaft den notwendigen Datenzugang zu erkämpfen.

Daten sind zentral für die wissenschaftliche Forschung. In naturwissenschaftlichen und technischen Fächern ist dies seit der Renaissance unbestritten. In den Sozial- und Wirtschaftswissenschaften gewinnt die empirische Forschung in den letzten Jahrzehnten immer mehr an Bedeutung und damit steigt der Bedarf an Daten. Gerade wenn es dort um repräsentative Aussagen und um Kausalitäten geht, ist eine entsprechende Datengrundlage unumgänglich. Aber auch Disziplinen, die bisher weitgehend hermeneutisch-qualitativ gearbeitet haben, werden sich in Zukunft wohl verstärkt mit datengestützten Methoden auseinandersetzen müssen. Ablesbar ist der Trend zur Quantifizierung etwa an der rezenten Häufung von Professuren mit dem Präfix „Daten-“ und dem Entstehen neuer Institute und Forschungsgruppen, die sich mit Big Data befassen. ${ }^{1}$ Hinter dieser Entwicklung stehen nicht zuletzt die Politik und die öffentliche Verwaltung, die regelmäßig die Ergebnisse datengetriebener Forschung nachfragen und entsprechende Studien beauftragen, um mit den Resultaten politische und administrative Maßnahmen zu initiieren und zu rechtfertigen. Ironischerweise sind in Österreich die Politik und die öffentliche Verwaltung gleichzeitig auch der größte Stolperstein, wenn es darum geht, der Wissenschaft dringend benötigte Datenbestände zugänglich zu machen. Während an- 
dere Länder, insbesondere in Skandinavien, innerhalb des europäischen Rechtsrahmens Wege gefunden haben, Register- und Statistikdaten für die Wissenschaft zu öffnen, scheitern derartige Initiativen in Österreich regelmäßig. Über die Hintergründe lässt sich nur spekulieren. Befürchtungen, mit Forschungsergebnissen konfrontiert zu werden, die der eigenen politischen Agenda zuwiderlaufen, dürften dabei aber eine erhebliche Rolle spielen.

\section{Demokratische Kontrolle und gesellschaftlicher Nutzen}

Für eine effektive demokratische Kontrolle von Regierung und öffentlicher Verwaltung wäre es natürlich notwendig, politische Maßnahmen und ihre Konsequenzen auf der Basis von Daten durch die Wissenschaft analysieren zu können. Dies wäre ein unschätzbarer Beitrag zu einem rationalen öffentlichen Diskurs zu wichtigen politischen Themen. So werden beispielsweise jedes Jahr viele hunderte Millionen Euro Steuergeld mittels der sogenannten „Forschungsprämie“ an österreichische Unternehmen ausgeschüttet, ohne zu wissen, ob diese Mittel auch den erwünschten Erfolg zeitigen, nämlich die Fördernehmer zu mehr Forschung anzuregen und damit zusätzliche Innovationsleistungen auszulösen. Um herauszufinden, ob die „Forschungsprämie“ ihre Aufgabe erfüllt, oder ob sie wirkungslos verpufft, müssten die vorliegenden Datenbestände analysiert werden. Der Zugang zu diesen bleibt der Wissenschaft aber bisher verwehrt. Darüber hinaus benötigt die Verwaltung aber auch abseits größerer politischer Diskussionen datenbasierte wissenschaftliche Analysen, um politische Vorgaben operativ umsetzen und dabei begleitend überprüfen zu können. Denn die im Zuge des Verwaltungshandelns angefallenen Daten geben in der Regel eine sehr komplexe Wirklichkeit wieder, sodass es oft die Hilfe der wissenschaftlichen Forschung braucht, um valide und belastbare Aussagen abzuleiten.

Die Wissenschaft benötigt allerdings nicht nur Zugang zu jenen Datenbeständen, die im Rahmen des Verwaltungshandelns anfallen, im Fachjargon „Registerdaten“ genannt, sondern auch zu jenen, die von der amtlichen Statistik erhoben werden, sogenannte Statistikdaten, und die in wesentlichen Teilen für die Wissenschaft verschlossen bleiben. Dabei geht es nicht um tabellarische Auswertungen oder Grafiken, sondern - wie auch bei den Registerdaten - um große Datenbestände auf Einzelfallebene (Mikrodaten). Während die Registerdaten verstreut bei den einzelnen Trägern der Verwaltung liegen, gibt es für die Statistikdaten eine Stelle, die alle Datenbestände zentral verwaltet, die Bundesanstalt Statistik Austria. Dazu 
gehören zum Bespiel umfangreiche demographische Untersuchungen oder auch Erhebungen bei Unternehmen, etwa zur Produktionstätigkeit, zu Innovationen und zur Digitalisierung. Zahlreiche Statistikdaten kommen auch aus der öffentlichen Verwaltung, sodass hier eine gewisse Überschneidung mit den Registerdaten der öffentlichen Hand besteht.

Die gute Nachricht lautet also: Es gibt im Bereich der öffentlichen Verwaltung und der amtlichen Statistik zahlreiche und sehr umfangreiche Datenbestände, deren wissenschaftliche Analyse evidenzbasiertes politisches und administratives Handeln zum Vorteil der gesamten Gesellschaft ermöglichen würde. Diese Datenbestände könnten auch zusammengeführt werden und so zu umfassenden Erkenntnissen führen, die bisher undenkbar schienen. So würde eine Verknüpfung der bereits erwähnten Daten zur Forschungsprämie mit statistischen Erhebungen zur Forschungstätigkeit und Daten zu Investitionen, weiteren Förderungen und zur Struktur der Belegschaft die Möglichkeit bieten, völlig neue Einsichten in das österreichische Wirtschaftssystem zu erlangen und es der Politik erlauben, zielgerichtet Maßnahmen zur Erhaltung des Wirtschaftsstandortes zu setzen, den Sozialstaat abzusichern und milliardenschwere Fehlinvestitionen zu vermeiden.

Im Bildungsbereich könnte etwa erforscht werden, wie sich pädagogische Modelle auf Bildungskarrieren und damit auch auf Arbeitsmarktchancen auswirken. Hier sind langfristige Datensammlungen sehr wichtig, weil der Erfolg oder Misserfolg von Maßnahmen sich manchmal erst Jahrzehnte später manifestiert und es fast unmöglich ist, die erforderlichen Daten rückwirkend zu erheben. Diese Daten sind aber nicht nur für die nachträgliche Evaluierung wichtig, sondern insbesondere auch ex ante - bevor Maßnahmen beschlossen werden. Dabei kann man auf Datenbestände zurückgreifen, die in Modell- und Schulversuchen gewonnen wurden. Gerade in Bereichen wie der Bildung, in der ideologische Einstellungen in der Politik und der Öffentlichkeit eine große Rolle spielen, ist es zentral, Maßnahmen auf Evidenzen aufzubauen, auch und gerade dann, wenn sie nicht in das jeweilige Weltbild der Entscheidungsträger*innen passen. Denn ist ein Lösungsansatz erst einmal etabliert, sind seine Konsequenzen nur mehr unter erheblichem Aufwand - und im Bildungsbereich oft gar nicht mehr - korrigierbar.

Ein weiteres Beispiel sind Krankenversicherungsdaten und andere Daten des Gesundheitswesens. Die Analyse dieser Datenbestände kann helfen, künftiges menschliches Leid zu lindern sowie die Kosten des Gesundheitssystems - etwa durch zielgerichtete präventive Maßnahmen - zu reduzieren. Epidemiologische Untersuchungen können Hinweise auf versteckte Krankheitsursachen liefern und so helfen, wirksame Therapien zu entwickeln, z.B. in der Krebsnachsorge oder bei psychischen Erkrankungen. 


\section{Zerklüftete Rechtslage}

Damit kommen wir zur schlechten Nachricht: Während vor allem die skandinavischen Länder Dänemark und Schweden, aber auch die Niederlande bei der wissenschaftlichen Nutzung von Register- und Statistikdaten weit fortgeschritten sind, liegt Österreich hier abgeschlagen zurück. ${ }^{2}$ Auch Deutschland und Frankreich sind hier bereits deutlich weiter ${ }^{3}$. Zwar kommt es schon heute in Österreich vereinzelt und anlassbezogen zur wissenschaftlichen Nutzung von Registerdaten. Grundlage dafür sind allerdings nur einzelvertragliche Vereinbarungen zwischen Forschungseinrichtungen und den Verwaltungsstellen, die über die Registerdaten verfügen. Damit obliegt es der Verwaltung und der ihr übergeordneten Politik, darüber zu entscheiden, wer im Einzelfall zu welchem Zweck mit Registerdaten forschen darf; oder eben auch nicht. Dies ist natürlich aus dem Blickwinkel der Unabhängigkeit der Wissenschaft und der demokratischen Kontrolle von staatlichem Handeln nicht akzeptabel.

Große Hoffnungen ruhen auf dem Forschungsorganisationsgesetz, ${ }^{4}$ das 2018 dahingehend novelliert wurde, der Wissenschaft endlich einen umfassenden Zugang zu Registerdaten zu ermöglichen und dafür zu sorgen, dass auch Daten aus unterschiedlichen Registern miteinander verknüpft werden können, um so die Aussagekraft der Daten massiv zu steigern. Betrieben wurde diese Novelle vom zuständigen Ressort, dem Bundesministerium für Bildung, Wissenschaft und Forschung (BMBWF) mit großer Verve. Im politischen Prozess mussten aber Zugeständnisse an andere Ressorts gemacht werden. So gibt es nunmehr zwar einen Rechtsanspruch auf den wissenschaftlichen Zugang zu Registerdaten, sofern strikte Datenschutzmaßnahmen eingehalten werden, aber nur, wenn der/die jeweils inhaltlich zuständige Bundesminister*in gemeinsam mit der/dem Wissenschaftsminister*in die Daten per Verordnung freigegeben haben ${ }^{5}$. Somit entscheidet letztlich wieder die Politik, welche Daten für wissenschaftliche Analysen herangezogen werden dürfen, und welche nicht. Eine erste Verordnung, die dann auch als Muster für weitere Verordnungen dienen soll, ist im Entstehen und auch an den technischen und institutionellen Rahmenbedingungen für den wissenschaftlichen Datenzugang wird im BMBWF mit Nachdruck gearbeitet. Vom Forschungsorganisationsgesetz aus verfassungsrechtlichen Gründen nicht erfasst sind jedoch etwa Register, die landesgesetzlich verankert sind. Ob die Bundesländer jemals bereit sein werden, ihre Register auf gesetzlicher Basis für die Wissenschaft zu öffnen, steht völlig in den Sternen. 
Noch vertrackter ist die Lage hinsichtlich der Statistikdaten auf Individualebene. Das Bundesstatistikgesetz ${ }^{6}$ unterbindet den Zugang der Wissenschaft zu personen- und unternehmensbezogenen Daten, also wenn individuelle Personen oder Unternehmen identifiziert werden könnten. Nun liegt es aber in der Natur solcher Daten, dass sie sich nicht vollständig anonymisieren lassen. Auch wenn Namen und Adressen gesetzeskonform durch nicht rückführbare Kennnummern ersetzt werden, wird es immer Einzelfälle geben, die identifizierbar bleiben, weil sie über Alleinstellungsmerkmale verfügen. Seit einem Jahrzehnt bemüht sich die Wissenschaft, gemeinsam mit progressiven Kräften in der Bundesstatistik, die Politik zu einer Regelung zu bewegen, die das Bedürfnis der Wissenschaft nach Datenzugang mit dem Anspruch der erfassten Menschen und Unternehmen auf Datenschutz und Wahrung des Statistikgeheimnisses in Einklang bringt. Bisher sind alle diese Initiativen gescheitert, zuletzt im Juli 2019 im Nationalrat. Während sich die österreichische Politik also bei den Registerdaten dazu durchringen konnte, sie prinzipiell und unter Wahrung strikter Vorgaben zum Datenschutz der Wissenschaft zur Verfügung zu stellen, scheut sie diesen Schritt im Bereich der Statistikdaten noch. Die Bemühungen, hier eine Lösung zu finden, laufen weiter. Der Ausgang ist aber noch ungewiss. Dass eine DSGVO-konforme Lösung möglich ist, zeigen die skandinavischen Länder, die einen umfassenden Zugang für die Forschung bereits ermöglicht haben.

Im - aus wissenschaftlicher Sicht - Idealfall steht am Ende der Bemühungen um Zugang zu Register- und Statistikdaten ein Datenzentrum, in dem alle freigegebenen Register und alle Statistikdaten für die Wissenschaft zugänglich sind und miteinander verknüpft werden können. Ein solches „Austrian Micro Data Center" könnte etwa bei Statistik Austria als neue Abteilung oder Tochtergesellschaft angesiedelt werden. Damit würden unnötige Parallelstrukturen vermieden werden und Österreich könnte zu einem internationalen Spitzenreiter in der Forschung mit Register- und Statistikdaten aufsteigen. Damit würde der Wissenschaftsstandort Österreich massiv an Attraktivität gewinnen und wesentlich interessanter für internationale anerkannte Wissenschaftler*innen werden.

\section{Datenschutz als zentrales Anliegen}

Bei allen Bemühungen, der Wissenschaft den Zugang zu Register- und Statistikdaten zu gewähren, ist die Wahrung des Datenschutzes ein zen- 
trales Anliegen. Dazu gehört unter anderem, dass Merkmale, die eine direkte Identifikation erlauben, etwa Name und Adresse, aus den Daten entfernt und durch eine nicht rückführbare Kennzahl ersetzt werden, bevor die Wissenschaft Zugang erhält. Das Forschungsorganisationsgesetz schreibt dies für Registerdaten vor und auch alle Vorschläge zur Reform des wissenschaftlichen Zugangs zu Statistikdaten setzen solche Maßnahmen voraus. Dennoch kann es vorkommen, dass einzelne Personen oder Unternehmen aufgrund besonderer Merkmale identifizierbar bleiben. Daher sehen die Regelungen zum wissenschaftlichen Zugang zu Registerund Statistikdaten auch immer massive Auflagen zum Datenschutz und zur Datensicherheit vor. Wer dagegen verstößt, verliert nicht nur seine wissenschaftliche Reputation und den weiteren Zugang zu Daten, sondern sieht sich auch erheblichen Strafen gegenüber. Bei Verstößen gegen das Forschungsorganisationsgesetz können bspw. verwaltungsstrafrechtlich je nach Fallkonstellation die Strafbestimmungen der Datenschutzgrundverordnung zur Anwendung kommen und damit ein Strafrahmen von bis zu 20 Millionen Euro verhängt werden, während Verstöße gegen das Statistikgeheimnis im Bundesstatistikgesetz nach allen vorliegenden Entwürfen von Strafgerichten als Amtsmissbrauch sanktioniert würden. Verstöße gegen den Datenschutz hätten also enorme Folgen, und dies nicht nur verwaltungsstraf- sondern unter Umständen auch schadenersatzrechtlich.

Datenschutz bedeutet nicht, dass das Verarbeiten von Daten, die sich auf identifizierbare Personen beziehen, generell untersagt wäre, sondern dass es zu einer Interessenabwägung kommt. Im konkreten Fall steht das Interesse einzelner Personen und Unternehmen, nicht identifiziert zu werden, dem Interesse der Öffentlichkeit an den Ergebnissen wissenschaftlicher Forschung und ihrer zugrundeliegenden Daten gegenüber, die erforderlich sind, um der Politik und der Öffentlichkeit evidenzbasierte Entscheidungen zu ermöglichen, die der gesamten Gesellschaft zum Vorteil gereichen. Es geht aber auch um eine Risikoabwägung: Wie wahrscheinlich und wie groß ist ein möglicher Schaden im Vergleich zum potentiellen Nutzen? Da die Wissenschaft beim Umgang mit Register- und Statistikdaten grundsätzlich sehr strikte Maßnahmen zum Schutz der Personen und Unternehmen, die hinter den Daten stehen, einhalten muss, kann eine Interessenabwägung - insofern die Frage nach dem Nutzen vorab eindeutig bejaht wurde - eigentlich nur zu dem Ergebnis führen, dass der Wissenschaft ein möglichst umfangreicher Zugang zu Register- und Statistikdaten gewährt werden soll.

Mitteilungen der VÖB 72 (2019) Nr. 2: Open Science 


\section{Die Plattform Registerforschung}

Um die Interessen der wissenschaftlichen Community in den Lebens-, Sozial- und Wirtschaftswissenschaften in puncto Datenzugang gegenüber der Politik besser vertreten zu können, wurde 2018 - im Umfeld der Anpassung des Forschungsorganisationsgesetzes an die Datenschutzgrundverordnung - die Plattform Registerforschung ${ }^{7}$ gegründet. Die Plattform versteht sich als informelles Netzwerk von Expert*innen und Stakeholdern, die mit Registerdatenforschung befasst sind. Mittlerweile nehmen mehr als 100 Personen aus ganz Österreich am Netzwerk teil. Darüber hinaus wird die Initiative von 15 Institutionen unterstützt. Die Plattform soll die zentrale Ansprechstelle in puncto Zugang der Wissenschaft zu Registerdaten des öffentlichen Sektors für politische Akteur*innen, öffentliche Verwaltung und wissenschaftliche Einrichtungen sein. Durch das Netzwerk besteht die Möglichkeit, rasch die relevante Expertise im Bereich der Registerforschung zu aktivieren, um strukturiert und abgestimmt einschlägige Maßnahmen der Verwaltung und Gesetzesvorhaben zu begleiten und zu kommentieren. Die Plattform betreibt auch aktiv Öffentlichkeitsarbeit, um die Bedeutung der Registerforschung für Politik, Gesellschaft und Wissenschaft umfassend darzustellen.

„Die Politik bedeutet ein starkes langsames Bohren von harten Brettern mit Leidenschaft und Augenmaß zugleich“, schrieb Max Weber in „Politik als Beruf". ${ }^{8}$ Die Plattform Registerforschung nimmt diese Herausforderung an und wird weiter daran arbeiten, der österreichischen Wissenschaft den Zugang zu Register- und Statistikdaten zu ermöglichen. Andere Länder wie Dänemark, Schweden oder die Niederlande, die längst solche Möglichkeiten geschaffen haben, weisen hier den Weg. ${ }^{9}$ Dass Österreich hier massiv hinterherhinkt, ist ein nationales Versäumnis und ein hausgemachtes Problem, das auch den Bemühungen entgegen steht, das Land auf europäischer Ebene an die Innovation Leader heranzuführen. Erst wenn die Lebens- und Sozialwissenschaften regulären Zugang zu Register- und Statistikdaten haben, können sie ihr volles Potential zum Nutzen der Gesellschaft entfalten. 
Univ.Prof. Dr. Harald Oberhofer Wirtschaftsuniversität Wien, Institut für Internationale Wirtschaft / Österreichisches Institut für Wirtschaftsforschung (WIFO) E-Mail: harald.oberhofer@wu.ac.at

Mag. Gerhard Schwarz Österreichisches Institut für Wirtschaftsforschung (WIFO)

E-Mail: gerhard.schwarz@wifo.ac.at

Dr. Michael Strassnig ORCID: https://orcid.org/0000-0003-1395-0868 Wiener Wissenschafts-, Forschungs- und Technologiefonds (WWTF) E-Mail: michael.strassnig@wwtf.at

\section{Literatur}

Harald Badinger und Jesus Crespo Cuaresma (2019): Statistik Austria: Zeit für einen Neustart. Die Presse, 8.5.2019. https://diepresse.com/ home/meinung/gastkommentar/5624943/Statistik-Austria_Zeit-fuereinen-Neustart

Daniele Bega (2014): The HMRC Datalab: Sharing administrative and survey data on taxation with the research community, 61-74. https:// edoc.bbaw.de/files/2327/2014_FacingTheFuture_edoc.pdf

Stefan Bender, Anja Burghardt und David Schiller: International Access to Administrative Data for Germany and Europe, 75-86. https://edoc. bbaw.de/files/2327/2014_FacingTheFuture_edoc.pdf

Bericht der Bundesregierung an den Nationalrat gem. $\S 8$ (2) FOG über die Lage und Bedürfnisse von Forschung, Technologie und Innovation in Österreich (Forschungs- und Technologiebericht 2018). https://bmbwf.gv.at/ fileadmin/user_upload/forschung/FTB/ftb_2018_dt_BF_gesamt_bf.pdf

Bundesgesetz über allgemeine Angelegenheiten gemäß Art. 89 DSGVO und die Forschungsorganisation (Forschungsorganisationsgesetz FOG). https://www.ris.bka.gv.at/GeltendeFassung.wxe?Abfrage=Bund esnormen\&Gesetzesnummer $=10009514$

Bundesgesetz über die Bundesstatistik (Bundesstatistikgesetz 2000). https://www.ris.bka.gv.at/GeltendeFassung.wxe?Abfrage=Bundesnor men\&Gesetzesnummer $=10006095$

Roxanne Connelly, Christopher J. Playford, Vernon Gayle, Chris Dibben (2016): The role of administrative data in the big data revolution in 
social science research. Social Science Research 59, 1-12. https://doi. org/10.1016/j.ssresearch.2016.04.015

Jesus Crespo Cuaresma, Harald Oberhofer und Gerhard Schwarz (2018): Auf dem Datenfriedhof. Wiener Zeitung, 1.1.2018. https://www.wienerzeitung.at/meinung/gastkommentare/938417-Auf-dem-Datenfriedhof.html Max Weber (1919): Politik als Beruf. Duncker \& Humblot: München, Leipzig. http://www.nbn-resolving.org/urn/resolver.pl?urn=urn:nbn:de:ko bv:b4-32402-6

Matthew Woollard (2014): Administrative Data: Problems and Benefits. A perspective from the United Kingdom. In: Adrian Duşa, Dietrich Nelle, Günter Stock \& Gert G. Wagner (Eds.): Facing the Future - European Research Infrastructures for the Humanities and Social Sciences. SCIVERO Verlag: Berlin, 49-60. https://edoc.bbaw.de/files/2327/2014_FacingTheFuture_edoc.pdf

1 Gerade hier wird ein Mehrwert in der Nutzung administrativer Daten gesehen, vgl. Roxanne Connelly, Christopher J. Playford, Vernon Gayle, Chris Dibben (2016): The role of administrative data in the big data revolution in social science research. Social Science Research 59, 1-12. https://doi.org/10.1016/j.ssresearch.2016.04.015

2 Jesus Crespo Cuaresma, Harald Oberhofer und Gerhard Schwarz (2018): Auf dem Datenfriedhof. Wiener Zeitung, 1.1.2018. https:// www.wienerzeitung.at/meinung/gastkommentare/938417-Auf-demDatenfriedhof.html; Harald Badinger und Jesus Crespo Cuaresma (2019): Statistik Austria: Zeit für einen Neustart. Die Presse, 8.5.2019. https://diepresse.com/home/meinung/gastkommentar/5624943/ Statistik-Austria_Zeit-fuer-einen-Neustart

$3 \mathrm{Vgl}$. Bericht der Bundesregierung an den Nationalrat gem. § 8 (2) FOG über die Lage und Bedürfnisse von Forschung, Technologie und Innovation in Österreich (Forschungs- und Technologiebericht 2018). https:// bmbwf.gv.at/fileadmin/user_upload/forschung/FTB/ftb_2018_dt_ BF_gesamt_bf.pdf

4 Bundesgesetz über allgemeine Angelegenheiten gemäß Art. 89 DSG$\mathrm{VO}$ und die Forschungsorganisation (Forschungsorganisationsgesetz FOG). https://www.ris.bka.gv.at/GeltendeFassung.wxe?Abfrage=Bund esnormen\&Gesetzesnummer $=10009514$

5 Ibid. $\S 38$ b.

6 Bundesgesetz über die Bundesstatistik (Bundesstatistikgesetz 2000). https://www.ris.bka.gv.at/GeltendeFassung.wxe?Abfrage=Bundesnor men\&Gesetzesnummer $=10006095$ 
7 https://www.registerforschung.at/

8 Max Weber (1919): Politik als Beruf. Duncker \& Humblot: München, Leipzig., S. 66. http://www.nbn-resolving.org/urn/resolver.pl?urn=urn: nbn:de:kobv:b4-32402-6

9 Siehe auch Matthew Woollard: Administrative Data: Problems and Benefits. A perspective from the United Kingdom; Daniele Bega: The HMRC Datalab: Sharing administrative and survey data on taxation with the research community; Stefan Bender, Anja Burghardt und David Schiller: International Access to Administrative Data for Germany and Europe. Alle Beiträge sind im Konferenzband Adrian Duşa, Dietrich Nelle, Günter Stock \& Gert G. Wagner (Eds.) (2014): Facing the Future - European Research Infrastructures for the Humanities and Social Sciences. SCIVERO Verlag: Berlin enthalten. https://edoc.bbaw.de/files/2327/2014_FacingTheFuture_edoc.pdf 